J. Gerlici, I.O. Shvedchykova, J.A. Romanchenko, I.V. Nikitchenko

\title{
DETERMINATION OF THE RATIONAL GEOMETRICAL PARAMETERS OF PLATE TYPE ELEMENTS OF MAGNETIC MATRIX OF THE POLYGRADIENT SEPARATOR
}

Introduction. Polygradient magnetic separation has wide application in industry and in biomedicine. Working process in polygradient separators takes place in a matrix, magnetic elements of which create magnetic forces sufficient to remove small ferro- and paramagnetic inclusions. Problem. The influence of mutual arrangement of elements on character of distribution of magnetic field is not taken into account during calculation of characteristics of magnetic field in magnetic matrixes. It makes comparative analysis of matrixes of different configurations quite difficult. Fulfillment of comparative analysis of strength characteristics of magnetic fields of multicomponent matrixes of polygradient separators of various configurations requires further researches. Goal. To determine the dependence of the strength characteristics of the polygradient electromagnetic separator on the geometrical parameters of the plate type elements of the multicomponent matrix. Methodology. The finite element method for calculation of power characteristics of separator magnetic field, method of comparative analysis and simple search method for determination of rational geometric parameters of the matrix have been used during the solution of the paper problem. Results. Estimation of entire spectrum of force field in plane of working zones of investigated structures in twodimensional location for determination of rational variants of polygradient matrixes has been done. The main stages of computational experiment are given. Method of comparative analysis of power characteristics of investigated variants of matrix structures with corresponding characteristics of basic version of separator for determination of rational geometrical variants of polygradient matrixes has been applied. By results of calculations the rational geometric parameters of polygradient matrix has been chosen. The characteristics of power magnetic fields in working gaps of matrixes of polygradient separator have been studied. It made possible to determine the rational structural variants of matrix on basis of parameter of effective area of working zone. Practical value. The results of research can be used in practice of design of electromagnetic separators with polygradient matrixes. References 10, table 1, figures 4.

Key words: electromagnetic separator, polygradient matrix, inhomogeneity coefficient, working zone, criteria of geometric similarity.

Осуществлена оценка спектра силового поля в плоскости рабочих зон исследуемых структур полиградиентных матриц электромагнитного сепаратора в двумерной постановке. Приведены основные этапы вычислительного эксперимента. Для решения задачи по определению рациональных вариантов полиградиентных матриц был задействован метод сравнительного анализа силовых характеристик исследуемых вариантов структур матрицы с соответствующими характеристиками базового варианта сепаратора. Произведён выбор рациональных геометрических параметров пластинчатых элементов магнитной матрицы сепаратора по критерию эффективной площади рабочей зоны матрицы. Проведен сравнительный анализ полученных данных с результатами других исследователей. Библ. 10, табл. 1, рис. 4.

Ключевые слова: электромагнитный сепаратор, полиградиентная матрица, коэффициент неоднородности, рабочая зона, геометрические критерии подобия.

Introduction. Polygradient magnetic separation has been widely used in industry and in biomedicine. In polygradient separators, the working process takes place in a matrix, whose magnetized elements create magnetic forces sufficient for the removal of small ferro- and paramagnetic inclusions $[1,2]$.

In the practice of magnetic separation, at calculating the magnetic force $\boldsymbol{F}_{\boldsymbol{m}}$ acting on the body of the volume $V$, which is removed, most often they come out of expression [2]

$$
\boldsymbol{F}_{\boldsymbol{m}}=\mu_{0} \chi \boldsymbol{H} \operatorname{grad}(\boldsymbol{H}) V,
$$

where $\boldsymbol{H}$ is the magnetic field strength vector in the calculated area free of electric currents; $\mu_{0}$ is the magnetic constant; $\chi$ is the average magnetic susceptibility of the removed body which depends on its shape, the ratio of the size and the magnetic permeability of the substance.

From (1) it can be seen that the direction of the extraction force $\boldsymbol{F}_{\boldsymbol{m}}$ coincides with the direction of gradient $\operatorname{grad}(\boldsymbol{H})$ of the magnetic field strength $\boldsymbol{H}$. As a result, the specific force $\boldsymbol{f}_{\boldsymbol{m}}$ of the magnetic field of the separator is defined as the product of the magnetic field strength $\boldsymbol{H}$ on its gradient $\operatorname{grad}(\boldsymbol{H})$

$$
\boldsymbol{f}_{\boldsymbol{m}}=\boldsymbol{F}_{\boldsymbol{m}} /\left(\mu_{0} \chi V\right)=\boldsymbol{H} \operatorname{grad}(\boldsymbol{H}) \text {. }
$$

From (2) it follows that in order to obtain higher values of the extraction force $\boldsymbol{f}_{\boldsymbol{m}}$, it is necessary to increase the magnetic field strength $\boldsymbol{H}$ and its gradient $\operatorname{grad}(\boldsymbol{H})$. An increase in the magnetic field strength $\boldsymbol{H}$ in separators with electromagnetic excitation has its limit, due to the saturation of the elements of the magnetic circuit. The $\boldsymbol{H}$ growth is also associated with an increase in power consumption, which leads to a higher cost of devices. At the same time, increasing the values of $\operatorname{grad}(\boldsymbol{H})$ can be achieved by optimizing the shape, geometric sizes and the relative position of the elements of the multicomponent magnetic matrix of the separator. Research in this area represents the greatest practical and theoretical interest.

Analysis of literary data and problem definition. Analytical, numerical and experimental methods have been used to calculate the magnetic characteristics of polygradient separators. In [3], the influence of the shape of the intersection of the elements of the magnetic medium on the distribution of the magnetic flux density and the gradient of the magnetic field of the polygradient separator was studied. In this work, using the ANSYS software system implementing the finite element method,

(C) J. Gerlici, I.O. Shvedchykova, J.A. Romanchenko, I.V. Nikitchenko 
it has been established that the strongest and most heterogeneous magnetic field is provided by a polygradient medium based on triangular elements. In view of this, a large number of publications are devoted to studies of polygradient matrices based on triangular elements. Thus, in [4] the connection of the strength and gradient of the magnetic field around the acute angle of the magnetic matrix with the direction of the bisector of this angle with respect to the direction of the external field is substantiated. It is shown if the bisector of an acute angle is parallel to the direction of the field, then the strength and gradient of the field around the angle increase. In [5], it is shown that the width of the gaps between the triangular plates of the matrix should be 1.5-2 times larger than the maximum particle size, since reducing the gap width will lead to a rapid closure of the matrix. When choosing a tooth angle, pole height and plate height, the magnetic force and the zone of removal of inclusions should be taken into account [6].

The analysis of literary sources [1-6] has shown that the calculation of the magnetic field in the matrices of polygradient separators is carried out mainly in a limited volume of the working zone for single, in particular, triangular elements of the matrix, followed by the application of the resulting regularities to a group of elements. This does not take into account the influence of the relative arrangement of elements on the nature of the distribution of the magnetic field, which complicates the comparative analysis of the matrices of various configurations. Therefore, further studies of approaches to conducting a comparative analysis of the strength characteristics of magnetic fields of multicomponent matrices of polygradient separators of different configurations are required.

The goal of the work is the establishment of the dependence of the strength characteristics of the polygradient electromagnetic separator on the geometric parameters of the plate type elements of the multicomponent matrix.

Research material and results. In previous studies, the authors obtained the following results:

- an advanced design of a polygradient electromagnetic separator for cleaning bulk powder-like materials from fine-grained ferromagnetic impurities of $0.005-5 \mathrm{~mm}$ in size is proposed [7];

- six structural variants $S_{i}=\left(\mathrm{S}_{1}, S_{2}, \ldots, S_{6}\right)$ of a plate type magnetic matrix of a polygradient separator based on triangular elements, whose geometric models are shown in Table 1, were obtained using mirror, portable, central and sliding symmetry operations;

- using a computational experiment, a preliminary comparative analysis of $S_{i}$ structures was performed to evaluate the degree of magnetic field inhomogeneity in their working gaps [8].

The preliminary estimation of the degree of inhomogeneity of the magnetic field in the working zones of the synthesized structures is accomplished by comparing the indices of field inhomogeneity along characteristic lines [8] as which the lines joining the vertices of opposite triangular plates and passing through the regions with the highest non-uniformity of the magnetic field are taken. Along the characteristic lines in the working regions of the synthesized structures $S_{i}$, the local values of the magnetic field strength $H$ field were calculated using the tools of the Elcut program. After that, for the various configurations of the polygradient media, the coefficient $k_{i}$ of field heterogeneity was determined by the formula

$$
k_{i}=\left(H_{\max }-H_{\min }\right) /\left(H_{\max }+H_{\min }\right),
$$

where $H_{\max }, H_{\min }$ are the maximal and minimal values of the magnetic field strength, respectively.

Table 1

Geometric models of structural variants of the matrix

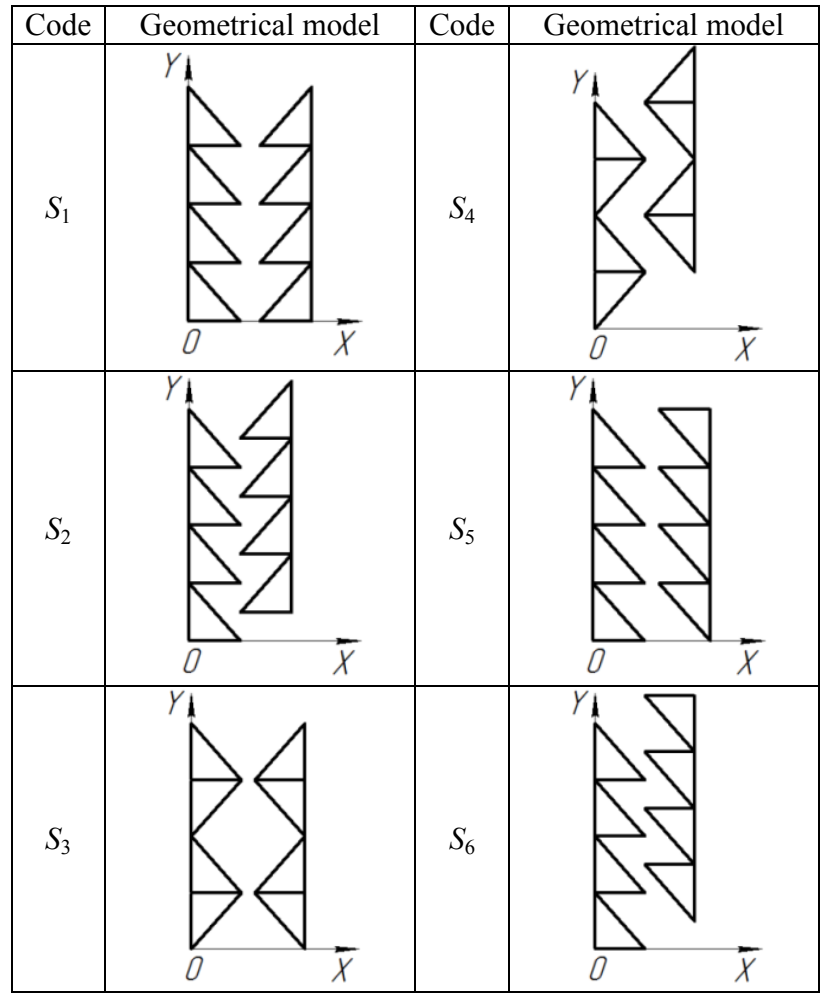

The main geometric dimensions of the working area, which varied in the study, are shown in Fig. 1 on an example of $S_{3}$ structure. Here are the notation: $\delta-$ the interpolar working gap which corresponds to the minimum distance between the plates; $\alpha$ - the angle at the vertex of the pole; $b$ - the base of the pole overhang; $a$ - the working width of the matrix. The following geometric similarity criteria for the studied areas were given: $X_{1}=b / a$ and $X_{2}=\alpha$. The ranges of variation of the geometric similarity criteria $X_{1}$ та $X_{2}$ of practical interest were: $X_{1}=b / a=0.1 \ldots 0.4 ; X_{2}=\alpha=0.11 \pi \ldots 0.44 \pi$.
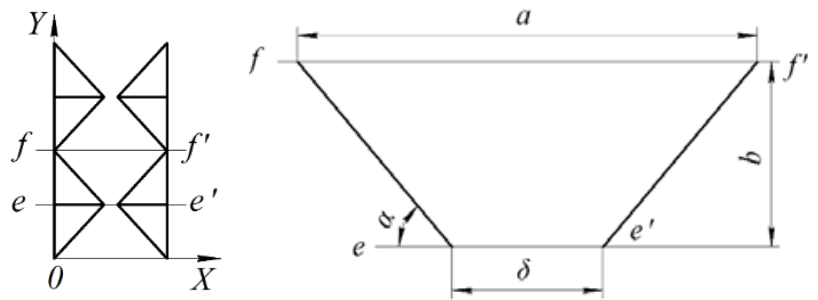

Fig. 1. Basic geometric dimensions of the working area on an example of the structure $S_{3}$ 
Calculated values of the heterogeneity coefficient $k_{i}$ varied from zero to one and were mapped according to the classification proposed in [9]: $k_{i} \leq 0.3$ - a weakly homogeneous (or slightly heterogeneous) field; $0.3<k_{i} \leq(0.9 \ldots 1)$ is a non-uniform field. This classification is in good agreement with the coefficient of variation $V$, the main statistical indicator characterizing the homogeneity of the data. In mathematical statistics, it is assumed that if the value of the coefficient $V$ is less than $33 \%$, then the aggregate of data is homogeneous, and if more than $33 \%$, then heterogeneous.

Thus, as a result of calculations, structures with weakly nonuniform fields for which $k_{i} \leq 0.3$ were cut off. Studies have also shown that in structures characterized by high inhomogeneity of the field, the average magnetic field strength $\boldsymbol{H}$ may be low. Therefore, at the next stage, for the purpose of determining rational variants of polygradient matrices, an estimate of the entire spectrum of the force field $\boldsymbol{H} \operatorname{grad}(\boldsymbol{H})$ in the plane of the working zones of the investigated structures in the twodimensional formulation was made. The experience of designing magnetic separators shows that the relative effect of a three-dimensional magnetic field is approximately the same for all points of plane magnetic models of working interpolar zones and does not depend on geometric similarity criteria $X_{1}=b / a$ and $X_{2}=\alpha$ [1]. Therefore, the magnetic field in the working zone of the separator can be considered planar parallel.

To solve the task of estimating the spectrum of the magnetic field, a program with the use of the Java 7 programming language and the Spring framework, which at the input of the input, processes the results of the calculation of the magnetic field strength $\boldsymbol{H}$, obtained in the Elcut program in the form of Excel files, was developed. Apache POI Library is intended to work with Excel files. The result of the program execution is a new Excel file with data of calculating the force characteristic $\boldsymbol{H} \operatorname{grad}(\boldsymbol{H})$ of the magnetic field. The main stages of the computational experiment are shown in Fig. 2.

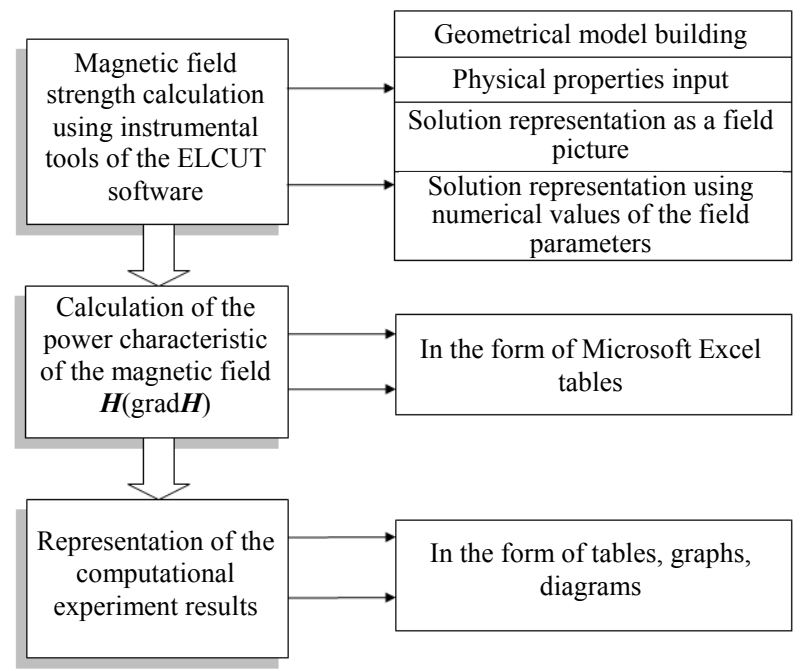

Fig. 2. Main stages of the computational experiment

To solve the problem of determining rational variants of polygradient matrices, a method of comparative analysis of force characteristics of investigated variants of matrix structures with corresponding characteristics of the basic variant of a separator, which is attributed to structure $S_{4}$, was used. The basic version of the magnetic separator was developed empirically and implemented in the form of a physical model, experimental studies of which confirmed its ability to operate [7]. The optimality test for the base version of the separator was not carried out. Characteristics of the basic separator model: structure code $S_{4}$; geometric similarity criteria $X_{1}=b / a=0.3$; $X_{2}=\alpha=0.11 \pi$.

For the base model of the separator, during the computational experiment, the part $P_{b}$ of the working zone (interpolar gap) of the matrix was determined, in which the power factor $f_{m}$ satisfies the requirement $f_{\min } \leq f_{m} \leq f_{\max }$, where $f_{\min }$ is the minimum limiting value of the force characteristic $f_{\text {min }}=(\boldsymbol{H} \operatorname{grad}(\boldsymbol{H}))_{\min }$, which provides, on the basis of the experience of designing magnetic separators, sufficient efficiency of removal of ferromagnetic inclusions (in the calculations the accepted value $\left.f_{\min }=3 \cdot 10^{8} \mathrm{~A}^{2} / \mathrm{m}^{3}[10]\right) ; f_{\max }$ is the maximum value of the specific reduced force obtained by calculation for the base model of the separator, which was in the research $f_{\max }=(\boldsymbol{H} \operatorname{grad}(\boldsymbol{H}))_{\max }=10.8 \cdot 10^{9} \mathrm{~A}^{2} / \mathrm{m}^{3}$. This part of the area of the working zone $P_{b}$ was expressed as a percentage relative to the value $P$ of the entire area of the working zone through the parameter $\gamma\left(\gamma=P_{b} / P\right)$. The parameter $\gamma$ depends on the geometric criteria $X_{1}, X_{2}$ and can be defined as the effective area of the working zone of the matrix. For the basic variant of the separator, the parameter $\gamma$ was $\gamma=7 \%$. In calculations, the parameter $\gamma_{1}$, which characterizes the part of the area of the working zone where the condition $f_{m}>f_{\max }=10.8 \cdot 10^{9} \mathrm{~A}^{2} / \mathrm{m}^{3}$ is fulfilled may also optionally be determined if necessary. Rationalities will be considered variants of the studied systems that satisfy the condition

$$
\gamma=\left(X_{1}, X_{2}\right) \rightarrow \max
$$

The results of the calculations obtained during the computational experiment showed that condition (3) best satisfies the structures $S_{4}$ and $S_{3}$ for which the highest values of the parameter $\gamma$ were obtained (Fig. 3). The maximum values of the parameter $\gamma$ for structures $S_{3}$ and $S_{4}$ were, respectively:

- at $X_{2}=\alpha=0.11 \pi-54.2 \%$ and $54.6 \%$;

- at $X_{2}=\alpha=0.18 \pi-60.3 \%$ and $65 \%$;

- at $X_{2}=\alpha=0.22 \pi-52.4 \%$ and $53.2 \%$;

- at $X_{2}=\alpha=0.28 \pi-42.1 \%$ and $42 \%$

- at $X_{2}=\alpha=0.33 \pi-32.0 \%$ and $31.6 \%$;

- at $X_{2}=\alpha=0.39 \pi-23.5 \%$ and $22.9 \%$.

The peculiarity of the structures $S_{4}$ and $S_{3}$ is that in both structures the single plate type element has the shape of an isosceles triangle, the direction of the bisector of an acute angle at the vertex of which coincides with the direction of the external magnetizing field. These results are in good agreement with the conclusions obtained in $[2,4]$, where it is established that the strength and gradient of the field around the angle of the triangular element of the matrix increase when the bisector of the acute angle is parallel to the direction of the field. 

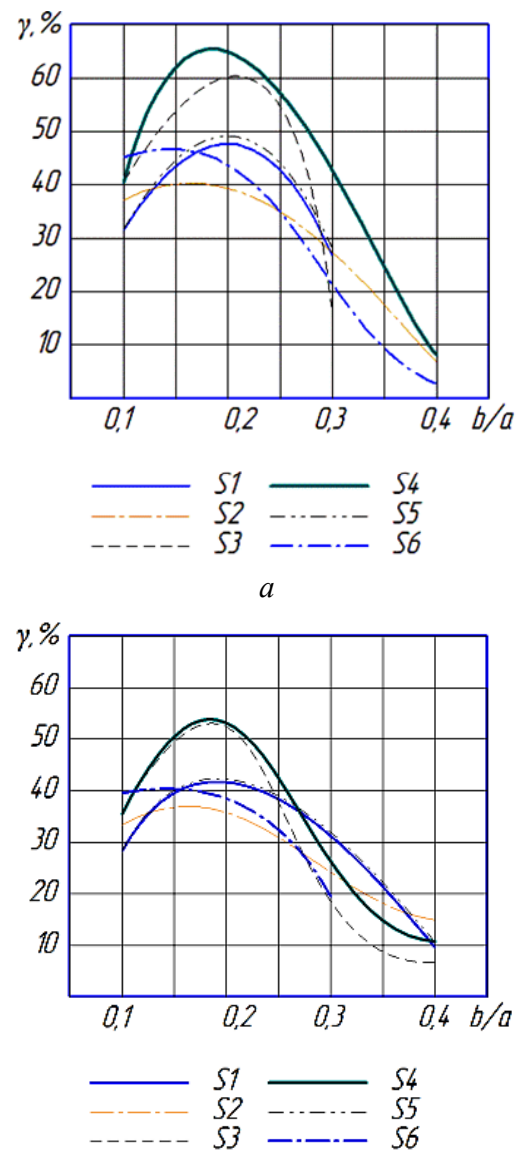

b
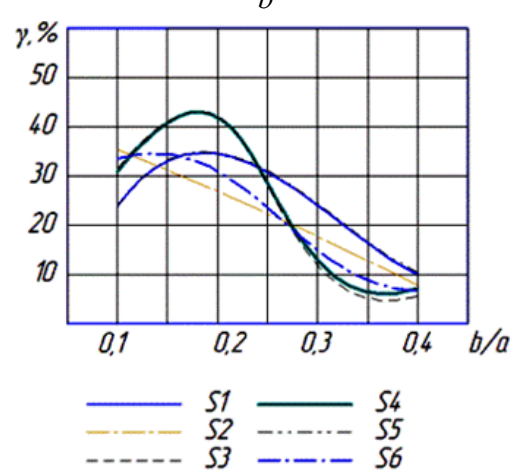

$c$
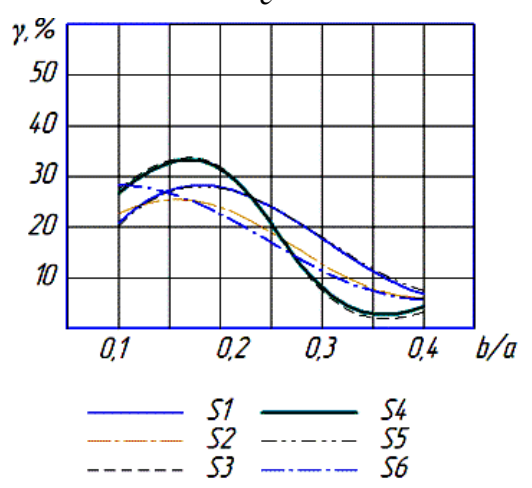

$d$

Fig. 3. Results of variant calculations of the parameter $\gamma$ : a) $\left.X_{2}=\alpha=0.18 \pi ; b\right) X_{2}=\alpha=0.22 \pi ; c$ ) $X_{2}=\alpha=0.28 \pi ; d$ ) $X_{2}=\alpha=0.33 \pi$

As data of Fig. 3 show, the parameter $\gamma(\gamma=65 \%)$ becomes the maximum value for the $S_{4}$ structure at $X_{1}=b / a=0.18$. Therefore, the structure $S_{4}$ was chosen for further research.
For more accurate determination of rational geometric parameters of plate type elements of the matrix of structure $S_{4}$, the dependence $\gamma=f\left(X_{2}\right)$ is constructed for $X_{1}=b / a=0.18$ (Fig. 4), which has a clearly expressed extremum corresponding to the point $X_{2}=\alpha=0.15 \pi$, for which $\gamma$ becomes $\gamma=73.3 \%$. Thus, the parameters $X_{1}=b / a=0.18$ and $X_{2}=\alpha=0.15 \pi$ should be considered as rational for the structure $S_{4}$ (accordingly, the angle at the vertex of the triangular element of the matrix is $2 \alpha=0.3 \pi$ ). Such a result is consistent with the data given in [6], where it is established that the magnetic strength and intensity of the magnetic field in the edge of the tooth tend to increase, when the angle $2 \alpha$ at the tooth top satisfies the condition $2 \alpha \leq 0.37 \pi$.

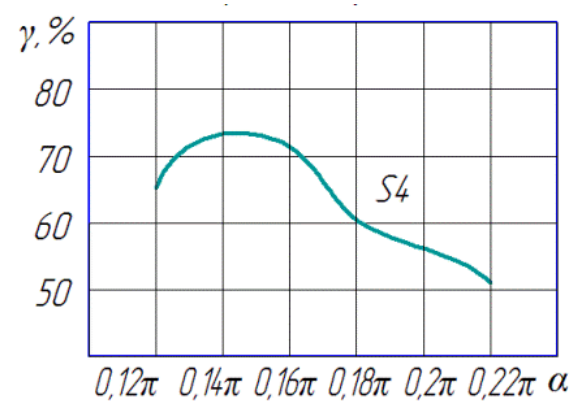

Fig. 4. Dependence of the parameter $\gamma=f\left(X_{2}\right)$ at $X_{1}=b / a=0.18$

\section{Conclusions.}

The dependence of the force characteristics of the polygradient electromagnetic separator on the geometric parameters of the plate type elements of the multicomponent matrix is established.

The analysis carried out in the work showed that the $S_{4}$ structure with the parameters $X_{1}=b / a=0.18$ and $X_{2}=\alpha=0.15 \pi$ can be considered rational in terms of the high value of the effective area of the working zone $(\gamma=73.3 \%)$. The results of the study are in agreement with the works of other authors.

\section{REFERENCES}

1. Zagirnyak M.V., Branspiz Yu.A., Shvedchikova I.A. Magnitnye separatory. Problemy proektirovanija [Magnetic separators. Problems of designing]. Kiev, Tehnika, 2011. 224 p. (Rus).

2. Ge W., Encinas A., Araujo E., Song S. Magnetic matrices used in high gradient magnetic separation (HGMS): A review. Results in Physics, 2017, vol.7, pp. 4278-4286. doi: 10.1016/j.rinp.2017.10.055.

3. Ren L., Zeng S., Zhang Y. Magnetic field characteristics analysis of a single assembled magnetic medium using ANSYS software. International Journal of Mining Science and Technology, 2015, vol.25, no.3, pp. 479-487. doi: 10.1016/j.ijmst.2015.03.024.

4. Song C.C., Ning G.H., Yuan Z.Y., Jing L.X., Hui C.C., Yao M.S. Investigation of the influence of different matrix rotation angles on the surrounding magnetic field in a uniform magnetic field. Ming Metall Eng, 2014, no.34, pp. 290-294.

5. Svoboda J. Magnetic Techniques for the Treatment of Materials. Boston, Kluwer Academic Publ., 2004, 99 p. doi: 10.1007/1-4020-2107-0.

6. Shun Z.Y., Liang S.C., Juan W.H., Yue W.F. Experimental study on magnetic separation by conical flux gathering media and optimization of its cone angle. Min Process Equip, 2012, pp. 74-79. 
7. Shvedchikova I.A., Lutsenko I.A., Romanchenko Ju.A. A study of polygradient media structure regularities. EasternEuropean Journal of Enterprise Technologies, 2015, vol.4, no.7(76), pp. 62-67. (Rus) doi: 10.15587/17294061.2015.47785.

8. Shvedchykova I., Romanchenko J., Nikitchenko I. Comparative analysis of inhomogeneity degree of magnetic field of polygradient magnetic separators for purification of bulk materials. 2017 International Conference on Modern Electrical and Energy Systems (MEES), Nov. 2017. doi: 10.1109/mees.2017.8248873.

9. Nikolov N.A. Quantitative criterion of the spatial inhomogeneity of the electromagnetic field in the near-field zone of a loop radiator. Cybernetics and Systems Analysis, 2013, vol.49, no.2, pp. 309-315. doi: 10.1007/s10559-013-9513-4.

10. Popov Yu.V. The examination practice of industrial safety of magnetic protection equipment (magnetic separators and columns) of production facilities for storage, processing and use of plant raw materials. Federal Service information bulletin, 2006, no.24, pp. 48-57. (Rus).
Juraj Gerlici ${ }^{1}$, Professor, Dr. Ing.,

I.O. Shvedchykova ${ }^{2}$, Doctor of Technical Sciences, Professor,

J.A. Romanchenko ${ }^{3}$, Lecturer,

I.V. Nikitchenko ${ }^{3}$, Senior Instructor,

${ }^{1}$ University of Žilina, Žilina, Slovak Republic,

1, Univerzitná, SK 01026 Žilina, Slovak Republic, phone 421(41)513 2550,

e-mail: juraj.gerlici@fstroj.uniza.sk

${ }^{2}$ Kyiv National University of Technologies and Design, 2, Nemirovich-Danchenko Str., Kyiv, 01011, Ukraine, phone +380509712574 ,

e-mail: ishved89@gmail.com

${ }^{3}$ Volodymyr Dahl East Ukrainian National University, 59-a, pr. Central, Severodonetsk, Lugansk region 93400 , Ukraine,

phone +380990326854,

e-mail: romanchenkojulia@i.ua

Received 16.04.2018

How to cite this article:

Gerlici J., Shvedchykova I.O., Romanchenko J.A., Nikitchenko I.V. Determination of the rational geometrical parameters of plate type elements of magnetic matrix of the polygradient separator. Electrical engineering \& electromechanics, 2018, no.4, pp. 58-62. doi: 10.20998/2074-272X.2018.4.10. 